\title{
Prognostic sensibility of clinical scales in Bell's Palsy: a prospective longitudinal study
}

\author{
Diane Picard ${ }^{1}$, Rémi Hervochon ${ }^{1}$, Elodie Lannadère ${ }^{1}$, Georges Lamas ${ }^{2}$, Frederic \\ TANKERE $^{2}$, and Peggy Gatignol ${ }^{3}$ \\ ${ }^{1} \mathrm{APHP}$ \\ ${ }^{2}$ Hopitaux Universitaires Pitie Salpetriere-Charles Foix \\ ${ }^{3}$ Sorbonne University
}

July 16, 2020

\begin{abstract}
Introduction: The first aim of the study was to compare three clinical scales at baseline: the Sunnybrook Facial Grading System (SFGS), the eFACE and the MBLF protocol. Then we intend to specify their sensibility to predict sequelae of Bell's palsy at 12 months. Methods: We carried out a longitudinal study of 23 patients with Bell's palsy. We reported every three months, from baseline to follow-up at 12 months, all the results of their facial assessment. In order to precise the criterion validity of the three clinical scales at baseline, effect of House and Brackmann severity grades was tested on SFGS composite score, eFACE dynamic score and MBLF total score. Then, these three scores were compared between group A (patients without sequelae at 12 months) and group B (patients with sequelae). Sensibility in predicting sequelae at 12 months was specified with Receiver Operating Characteristics curve for each clinical scale. Results: Grades effect on each clinical scale was found ( $\mathrm{p}<.005)$. At baseline, no significant difference was observed between group A and group B on clinical scales. At three months, only the MBLF total score was significant to predict recovery $\left(\chi^{2}=8.37, \mathrm{p}<.0038^{*}\right.$, AUC $\left.=.769\right)$. From the 6th month, the three clinical scales could predict sequelae at 12 month $(\mathrm{p}<.005)$. Conclusion: Clinical tools are essential to assess patients with Bell's palsy at baseline and follow-up. At baseline, the dynamic scores are not significantly different. At 3 months, only the MBLF significantly discriminated patients with or without sequelae at 12 months. At 6 months, all three scales were able to predict sequelae at 12 months.
\end{abstract}

\section{Hosted file}

BLINDED MANUSCRIPT.doc available at https://authorea.com/users/343429/articles/470071prognostic-sensibility-of-clinical-scales-in-bell-s-palsy-a-prospective-longitudinalstudy

\section{Hosted file}

TABLE.doc available at https://authorea.com/users/343429/articles/470071-prognosticsensibility-of-clinical-scales-in-bell-s-palsy-a-prospective-longitudinal-study

\section{Hosted file}

Table1.xlsx available at https://authorea.com/users/343429/articles/470071-prognosticsensibility-of-clinical-scales-in-bell-s-palsy-a-prospective-longitudinal-study

\section{Hosted file}

FIGURES.doc available at https://authorea.com/users/343429/articles/470071-prognosticsensibility-of-clinical-scales-in-bell-s-palsy-a-prospective-longitudinal-study 
figures/FIG1/FIG1-eps-converted-to.pdf 
figures/FIG2/FIG2-eps-converted-to.pdf 
figures/FIG3/FIG3-eps-converted-to.pdf 